\title{
BMJ Open Coverage and factors associated with full immunisation among children aged 12-59 months in Bangladesh: insights from the nationwide cross-sectional demographic and health survey
}

\author{
Abdur Razzaque Sarker, ${ }^{01,2}$ Raisul Akram, ${ }^{1}$ Nausad $A l i,{ }^{1}$ Marufa Sultana ${ }^{1}$
}

To cite: Sarker AR, Akram $\mathrm{R}$, Ali N, et al. Coverage and factors associated with full immunisation among children aged 12-59 months in Bangladesh: insights from the nationwide crosssectional demographic and health survey. BMJ Open 2019;9:e028020. doi:10.1136/ bmjopen-2018-028020

- Prepublication history for this paper is available online. To view these files, please visit the journal online (http://dx.doi. org/10.1136/bmjopen-2018028020).

Received 18 November 2018 Revised 9 May 2019 Accepted 31 May 2019

Check for updates

(c) Author(s) (or their employer(s)) 2019. Re-use permitted under CC BY-NC. No commercial re-use. See rights and permissions. Published by BMJ.

${ }^{1}$ International Centre for Diarrhoeal Disease Research Bangladesh, Dhaka, Bangladesh ${ }^{2}$ Bangladesh Institute of Development Studies, Dhaka, Bangladesh

Correspondence to Abdur Razzaque Sarker; razzaque@bids.org.bd

\section{ABSTRACT}

Objective To estimate the coverage and factors associated with full immunisation coverage among children aged 12-59 months in Bangladesh.

Study design The study is cross sectional in design. Secondary dataset from Bangladesh Demographic and Health Survey was used for this analysis. Immunisation status was categorised as 'fully immunised' if the children had received all the eight recommended vaccine doses otherwise 'partially/unimmunised'.

Settings Bangladesh.

Participant Children aged 12-59 months were the study participants. Participants were randomly selected through a two-stage stratified sampling design. A total of 6230 children were eligible for the analysis.

Results About $86 \%$ of the children (5356 out of 6230 ) were fully immunised. BCG has the highest coverage rate $(97.1 \%)$ followed by oral polio vaccine $1(97 \%)$ and pentavalent $1(96.6 \%)$, where the coverage rate was the lowest for measles vaccine (88\%). Coverage was higher in urban areas (88.5\%) when compared with rural ones (85.1\%). Full immunisation coverage was significantly higher among children who lived in the Rangpur division (adjusted OR (AOR) $=3.46 ; 95 \% \mathrm{Cl} 2.45$ to $4.88, \mathrm{p}<0.001$ ), were 48-59 months old ( $\mathrm{AOR}=1.32 ; 95 \% \mathrm{Cl} 1.06$ to 1.64 , $\mathrm{p}=0.013)$, lived in a medium size family ( $\mathrm{AOR}=1.56 ; 95 \%$ Cl 1.32 to 1.86, $p<0.001$ ), had parents with a higher level of education $(\mathrm{AOR}=1.96$; $95 \% \mathrm{Cl} 1.21$ to 3.17 , $\mathrm{p}=0.006$ and $\mathrm{AOR}=1.55 ; 95 \% \mathrm{Cl} 1.05$ to $2.29, \mathrm{p}=0.026$ ) and belonged to the richest families $(\mathrm{AOR}=2.2 ; 95 \% \mathrm{Cl}$ 1.5 to $3.21, p<0.001)$. The likelihood of being partially or unimmunised was higher among children who had the father as their sole healthcare decision-maker $(\mathrm{AOR}=0.69$; $95 \% \mathrm{Cl} 0.51$ to $0.92, \mathrm{p}<0.012$ ).

Conclusions There were significant variations of child immunisation coverage across socioeconomic and demographic factors. These findings will inform innovative approaches for immunisation programmes, and the introduction of relevant policies, including regular monitoring and evaluation of immunisation coverageparticularly for low-performing regions, so that the broader benefit of immunisation programmes can be achieved in all strata of the society.
Strengths and limitations of this study

- Our study did a pioneer investigation of full immunisation coverage across various regions in Bangladesh using the latest nationwide demography and health survey dataset.

- Multivariable logistic regression models were constructed to assess the potential factors associated with full immunisation coverage.

- The study results can be generalised at the country level because of its large sample size and using the latest nationally representative demographic and health survey data of Bangladesh.

- Due to the nature of the study, the possibility of recall bias with our results cannot be ignored.

\section{BACKGROUND}

Universal immunisation programme of children against vaccine-preventable diseases (VPD) has been recognised as one of the most cost-effective programmes to diminish childhood mortalities and morbidities across the world. ${ }^{12}$ Every year, vaccination against VPDs prevents debilitating illness and disability, saving millions of young lives globally. ${ }^{3}$ Over the decades, remarkable improvements have been made towards the development of national immunisation programmes, with the Expanded Program on Immunization (EPI) being a major contribution to this success. ${ }^{4}$ EPI was formally established in 1974 , with the support of $\mathrm{WHO}$, with the goal of immunising every child against six VPD (ie, diphtheria, pertussis, tetanus (DPT), poliomyelitis, measles and tuberculosis) by $1990 .^{5}$ The utmost priority was given to developing countries because of the higher prevalence and inadequate service delivery for immunisation within these settings. ${ }^{4}$ The government of Bangladesh had initiated the EPI with the support of Unicef and WHO through 
various outreach activities from 1979, with the overall objective to immunise all children by 1990 to prevent the VPDs and to eradicate poliomyelitis. ${ }^{56}$ Later, in 1995, national immunisation day was initiated by EPI to sustain the polio-free status and also to increase the immunisation coverage that was achieved through different operational activities. ${ }^{67}$ The implementation of the EPI has already been shown to be a great success globally. This can be seen through the significant improvement in child immunisation coverage and the eradication of poliomyelitis. ${ }^{8}$ In the line of success, Bangladesh has experienced impressive improvements in increasing immunisation coverage and a significant contribution to the reduction of childhood morbidity, mortality and also maintaining its polio-free status. ${ }^{9}{ }^{10}$ Despite the success in reducing the child mortality rate by two-thirds since 1990, Bangladesh recorded 0.1 million child deaths in $2016^{10}$ and was among the top 10 countries that had the highest childhood mortality globally. ${ }^{11}$ A recent study revealed that almost half of these deaths could be prevented through immunisation alone. ${ }^{3}{ }^{12}$ Therefore, a greater focus on the completion of all recommended EPI vaccines is needed to further achieve gains in decreasing childhood morbidities and mortalities. ${ }^{7}$ Unquestionably, improving the utilisation of routine immunisation services, and easy access to all vaccines is the best option to improve the immunisation coverage.

Although a number of studies have reported findings on child immunisation, few of them have generated evidence about the sociodemographic factors associated with full vaccination among children aged 12-59 months. ${ }^{13-15}$ Furthermore, available studies have either focused on specified geographical settings, age groups or ethnic groups rather than the nationwide setting and have not identified the determinants of individual vaccines separately. ${ }^{14-21}$ This study thus sought to capture the full immunisation status using the latest country representative Demographic and Health Survey (DHS) dataset. The DHS provides reliable information on individual-level immunisation coverage, as well as a range of factors that might influence immunisation practices. As such, the objective of this study was to estimate the extent of full immunisation coverage and to investigate the determinants of full immunisation using the nationally representative data of Bangladesh. Analysis of a nationally representative dataset would thus allow for a generation of the current evidence of vaccination practices that could be useful for international comparison and will help in implementing the relevant immunisation policies and priorities for the betterment of the child's health against VPD in Bangladesh.

\section{METHODS}

\section{Study design and population}

This study was based on a secondary analysis of the latest Bangladesh Demographic and Health Survey (BDHS) 2014 dataset. The DHS is a nationally representative cross-sectional survey, which uses a two-stage stratified sampling design to cover the target population of the entire country. This survey used the sampling frame provided by the Bangladesh Bureau of Statistics. This survey was carried out for 6 months, from June 2014 to November 2014. The BDHS 2014 used three types of questionnaires: household questionnaire, woman's questionnaire and community questionnaire. Through the women's questionnaire, up-to-date information on sociodemographic, maternal and child health indications including individual-level vaccination coverage was collected. Participants of this study were children aged between 1 and 5 years. Childhood immunisation history was collected for all surviving children over the last 5 years. Immunisation data were collected mainly from the records on vaccine cards; if vaccine card was not available, mothers were asked to recall about the vaccination history of the respective child. Face-to-face interviews that were conducted with reproductive-aged women (15-49 years) were conducted for the collection of data using the structured questionnaire. ${ }^{22}$ Details about a sampling technique, survey design and quality control have been described elsewhere. ${ }^{23}$ All the DHS data were publicly accessible and were made available on request by the MEASURE DHS. Furthermore, approval was sought from and given by the MEASURE DHS programme office in the use of this dataset for this specific study. According to the DHS, written informed consent was obtained from all participants prior to the interviews.

\section{Outcome variable}

The outcome variable of the analysis was the children's immunisation status and categorised as fully immunised and partially/unimmunised. Vaccination status was categorised as 'fully immunised' if the children had received doses of all the eight recommended vaccines: one dose of the vaccine against tuberculosis (BCG), three doses of pentavalent (DPT, Haemophilus influenzae type B and HepB), three doses of oral polio vaccine (OPV) and one dose of measles vaccines. ${ }^{23}$ Children aged 12-59 months of age were included in this analysis to capture the vaccination status of the children. Children younger than 12 months of age were excluded as they were not old enough to receive the full schedule of the routine vaccines. WHO recommended vaccination schedule for Bangladesh ${ }^{24}$ is presented in table 1. Immunisation histories for all vaccines were coded as dummy responses, with 1 for fully immunised and 0 otherwise. In a small number of cases, the immunisation cards were not available, and mothers responded with 'don't know' while asked about their children's vaccination status on certain vaccines. In that cases, the vaccination status of children was considered as 'not fully immunised' since such responses reflect a negative response regarding immunisation. ${ }^{14}$

\section{Explanatory variables}

Explanatory variables were selected based on the published literature, prior knowledge and availability of 
Table 1 The Expanded Program on Immunizations schedule in Bangladesh

\begin{tabular}{lll}
\hline Diseases & Name of vaccine & Recommended age \\
\hline Childhood tuberculosis & BCG & At birth/0 day \\
Diphtheria/tetanus/pertussis/hepatitis B/ & Pentavalent 1 & 42 days \\
Hib pneumonia and meningitis & Pentavalent 2 & 70 days \\
& Pentavalent 3 & 98 days \\
Poliomyelitis & OPV 1 & 42 days \\
& OPV 2 & 70 days \\
Measles & OPV 3 & 98 days \\
\hline
\end{tabular}

Hib, Haemophilus influenzae type B; OPV, oral polio vaccine.

variables in the BDHS 2014 dataset. Area of residence, administrative division, sex and age of children, birth order, family size, parental education, mass media exposure, household wealth index and the mother's decision-making ability for both the children and their own healthcare matters were included as explanatory variables. In this analysis, the categorisation of continuous variables was done in light of previous literature where the child's age was categorised into four groups at 12-month intervals, and maternal age was categorised into three groups (less than 20 years, 20-34 years and more than 34 years). Self-reported parental (both mother and father) educational attainment was used and categorised as 'no education', 'primary', 'secondary' and 'higher'. No education refers to there not having any formal education, with primary was defined as completing grade 5, secondary as completing grade 10 and higher was defined as attaining more than grade 10. Family size was determined by the number of family members who lived together in a household, and defined by the BDHS. Family size was categorised on the basis of other published literature and categorised as 'small', 'medium' and 'large'. A 'small' family size refers to the family consisting of less than four members, 'medium' as four to six members and 'large' if the family member exceeds six members. Mass media access was categorised as 'yes' if the family had access to televisions and radios and 'no' otherwise. We used the predetermined wealth index category provided in the dataset generated from selected household assets using principal component analysis and classified it into five groups, namely: 'poorest', 'poorer', 'middle', 'rich' and 'richest'. Moreover, both the decision-making ability of the mothers for both their children and personal healthcare were categorised into four groups as 'herself', 'jointly with husband', 'husband alone' and 'other'.

\section{Statistical analysis}

The original dataset comprised 8092 children, aged 0-59 months of age. In the case of our analysis, 1555 children were excluded as they were under the age of 12 months. Moreover, 307 cases of missing data were present, and these were cases where no information was provided (no responses, neither 'yes', 'no' nor 'don't know') on vaccination information, and thus also excluded from the analysis. Finally, a total of 6230 children were eligible and included in the analysis. A proper sampling weight was used in this analysis to make the sample more representative of the population at the national level. Descriptive statistics, such as frequency with percentages, were executed to represent the background characteristics of study participants, and a proportion with $95 \%$ CI was used for presenting the coverage rate of fully vaccinated children. The association between each independent variable and the full vaccination uptake was investigated using a univariate analysis. Multivariable logistic regression that adjusted for all the selected independent variables was also constructed to determine the significant influencing factors for fully vaccination and results were presented in terms of adjusted OR(s) (AOR) with a 95\% CI. Before the execution of a multivariate regression model, a bivariate analysis was conducted to trace out the significant factors for full immunisation. Variables significant at $\mathrm{p}$ $\leq 0.05$ were included in the multivariate logistic regression analysis. Variance inflation factor (VIF) was calculated to detect any multicollinearity in the model. The low value of average VIF (3.07) confirms no notable multicollinearity among variables. Linktest command through Stata/SE V.13.0 indicated that the constructed model was well specified where the Hosmer-Lemeshow test statistics of goodness of fit indicates the acceptance of the model. Data cleaning, validation and all statistical analyses were performed using Stata/SE V.13.0 (Stata Corporation).

\section{Patient and public involvement}

No patients were involved in developing the research question, outcome measure and design of the study. We are unable to disseminate the results of the research directly to study participants.

\section{RESULTS}

\section{Descriptive statistics}

Distribution of study participants across the sociodemographic variables is presented in table 2. The proportion 
Table 2 Baseline characteristics of study participants and their immunisation coverage in Bangladesh, $2014(n=6230)$

\begin{tabular}{lll}
$\begin{array}{l}\text { Characteristics of } \\
\text { sample }\end{array}$ & Frequency $(\%)$ & $\begin{array}{l}\text { Fully immunised } \\
\%(95 \% \mathrm{Cl})\end{array}$ \\
\hline
\end{tabular}

Immunisation status

Partially/unimmunised 874 (14.03)

Fully immunised $5356(85.97)$

Area of residence

\begin{tabular}{|c|c|c|}
\hline Urban & 1577 (25.32) & 88.50 (86.83 to 89.98$)$ \\
\hline Rural & 4653 (74.68) & 85.11 (84.06 to 86.10 ) \\
\hline \multicolumn{3}{|l|}{ Division } \\
\hline Rajshahi & $642(10.30)$ & 86.16 (83.26 to 88.62$)$ \\
\hline Barisal & $352(5.65)$ & 84.89 (80.75 to 88.27$)$ \\
\hline Chittagong & $1336(21.45)$ & 85.38 (83.38 to 87.18$)$ \\
\hline Dhaka & $2187(35.11)$ & 88.81 (87.42 to 90.07$)$ \\
\hline Khulna & $468(7.51)$ & 89.01 (85.84 to 91.55$)$ \\
\hline Rangpur & $624(10.01)$ & 91.48 (89.01 to 93.43$)$ \\
\hline Sylhet & $622(9.98)$ & 69.81 (66.08 to 73.30$)$ \\
\hline \multicolumn{3}{|l|}{ Sex } \\
\hline Male & $3236(51.94)$ & 85.57 (84.31 to 86.73 ) \\
\hline Female & 2994 (48.06) & 86.40 (85.13 to 87.58$)$ \\
\hline
\end{tabular}

Age category (in

months)

\begin{tabular}{|c|c|c|}
\hline $12-23$ & $1630(26.17)$ & 83.97 (82.10 to 85.67$)$ \\
\hline $24-35$ & $1560(25.04)$ & 86.68 (84.90 to 88.28$)$ \\
\hline $36-47$ & $1529(24.55)$ & 86.63 (84.83 to 88.24$)$ \\
\hline $48-59$ & $1510(24.24)$ & 86.72 (84.91 to 88.34$)$ \\
\hline \multicolumn{3}{|l|}{ Birth order } \\
\hline 1 & 2346 (37.66) & 88.35 (86.99 to 89.59$)$ \\
\hline 2 & $1880(30.18)$ & 87.66 (86.09 to 89.07$)$ \\
\hline$\geq 3$ & 2003 (32.16) & 81.59 (79.83 to 83.22$)$ \\
\hline \multicolumn{3}{|l|}{ Mother's age } \\
\hline$<20$ years & $690(11.07)$ & 86.85 (84.12 to 89.18$)$ \\
\hline 20-34 years & $5011(80.43)$ & 85.97 (84.98 to 86.91$)$ \\
\hline 35 years and more & $529(8.50)$ & 84.75 (81.43 to 87.57$)$ \\
\hline \multicolumn{3}{|l|}{ Family size (members) } \\
\hline Small $(<4)$ & $772(12.39)$ & 86.35 (83.74 to 88.59$)$ \\
\hline Medium (4-6) & 3609 (57.93) & 87.88 (86.77 to 88.90$)$ \\
\hline Large $(>6)$ & $1849(29.68)$ & 82.08 (80.26 to 83.76$)$ \\
\hline \multicolumn{3}{|l|}{ Mother's education } \\
\hline No education & $1053(16.91)$ & 76.77 (74.13 to 79.23$)$ \\
\hline Primary & $1740(27.93)$ & $81.17(79.27$ to 82.94$)$ \\
\hline Secondary & $2870(46.07)$ & 90.62 (89.50 to 91.64$)$ \\
\hline Higher & 566 (9.09) & 94.22 (91.97 to 95.87$)$ \\
\hline \multicolumn{3}{|l|}{ Father's education } \\
\hline No education & $1683(27.02)$ & 78.64 (76.61 to 80.53$)$ \\
\hline Primary & $1843(29.58)$ & 84.84 (83.13 to 86.41$)$ \\
\hline Secondary & $1857(29.81)$ & 90.21 (88.77 to 91.48 ) \\
\hline Higher & $847(13.60)$ & 93.69 (91.83 to 95.14$)$ \\
\hline Mass media access & & \\
\hline
\end{tabular}

Continued

\begin{tabular}{|c|c|c|}
\hline $\begin{array}{l}\text { Characteristics of } \\
\text { sample }\end{array}$ & Frequency (\%) & $\begin{array}{l}\text { Fully immunised } \\
\%(95 \% \mathrm{Cl})\end{array}$ \\
\hline Yes & $2546(40.87)$ & 90.62 (89.42 to 91.69$)$ \\
\hline No & $3684(59.13)$ & 82.75 (81.50 to 83.94$)$ \\
\hline \multicolumn{3}{|l|}{ Wealth index } \\
\hline Poorest & $1438(23.08)$ & 76.13 (73.85 to 78.26$)$ \\
\hline Poorer & 1160 (18.62) & 85.32 (83.16 to 87.24$)$ \\
\hline Middle & 1184 (19.01) & 89.07 (87.17 to 90.73$)$ \\
\hline Richer & $1260(20.23)$ & 88.10 (86.20 to 89.78$)$ \\
\hline Richest & $1187(19.05)$ & 93.16 (91.58 to 94.46$)$ \\
\hline \multicolumn{3}{|l|}{$\begin{array}{l}\text { Child's healthcare } \\
\text { decision-maker }\end{array}$} \\
\hline Herself & $934(15.25)$ & 88.08 (85.84 to 90.01$)$ \\
\hline Jointly with husband & $3577(58.37)$ & 87.94 (86.84 to 88.97 ) \\
\hline Husband alone & 1249 (20.38) & 79.08 (76.73 to 81.25$)$ \\
\hline By other & $368(6.00)$ & 88.30 (84.59 to 91.21$)$ \\
\hline \multicolumn{3}{|l|}{$\begin{array}{l}\text { Mother's healthcare } \\
\text { decision-maker }\end{array}$} \\
\hline Herself & 764 (12.46) & 89.23 (86.82 to 91.24$)$ \\
\hline Jointly with husband & 3208 (52.33) & 87.91 (86.74 to 88.99 ) \\
\hline Husband alone & $1797(29.32)$ & 81.78 (79.93 to 83.50$)$ \\
\hline By other & $361(5.88)$ & 86.23 (82.27 to 89.42$)$ \\
\hline
\end{tabular}

of full vaccination was $86 \%$ (5356 out of 6230 children). Among the participants, $74.7 \%$ were from rural areas and $35.1 \%$ were from the Dhaka division. Participants were almost equally distributed by sex and age categories. Among the participants, $37.7 \%$ was the first-born child, and most of the children's families $(57.9 \%)$ consisted of four to six members. Majority of the mothers (80.4\%) were aged between 20 and 34 years. $46.1 \%$ of the mothers and $29.8 \%$ of fathers had secondary-level education. Less than half of the mothers $(40.9 \%)$ had access to mass media, for example, radio and television. In addition, a majority of the mothers reported that they had the capacity for decision-making with regards to both their own as well as their children's healthcare.

\section{Vaccination coverage rate among study participants}

Full immunisation coverage rate of study participants is also presented in table 2. Findings revealed that full immunisation coverage was slightly higher in urban areas $(88.5 \%)$ as compared with rural areas $(85.1 \%)$ and was found to be the highest in the Rangpur division $(91.5 \%)$ followed by Khulna (89\%), Dhaka $(88.8 \%)$ and the lowest in Sylhet division (69.8\%). The immunisation coverage across individual vaccines is shown in figure 1 . Full immunisation coverage was similar across sex and age categories of the children. However, first-born child had slightly higher vaccine coverage rates $(88.4 \%)$ than the other $87.7 \%$ for second and $81.6 \%$ for third or more birth order). 

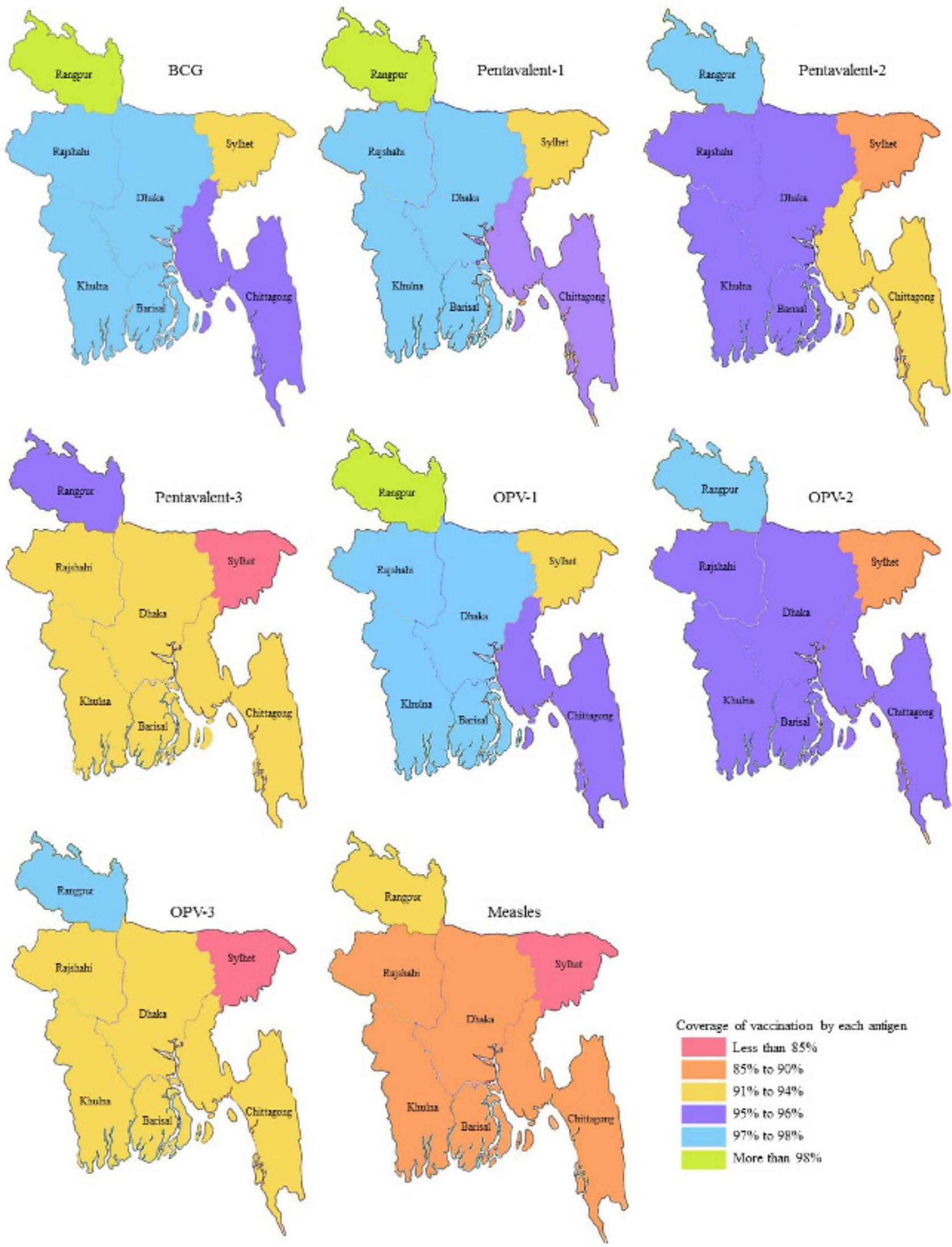

\section{Coverage of vaccinstion by each sutigen}

Less than $85 \%$ $85 \%$ to $90 \%$ $91 \%$ to $94 \%$ $95 \%$ to $95 \%$ $97 \%$ to $99 \%$ More than $98 \%$

Map of Bangladesh by administrative division

Figure 1 Coverage rate by individual vaccines (BCG, pentavalent, oral polio vaccine (OPV) and measles) across administrative divisions in Bangladesh (map obtained from open access source and edited according to study findings). 


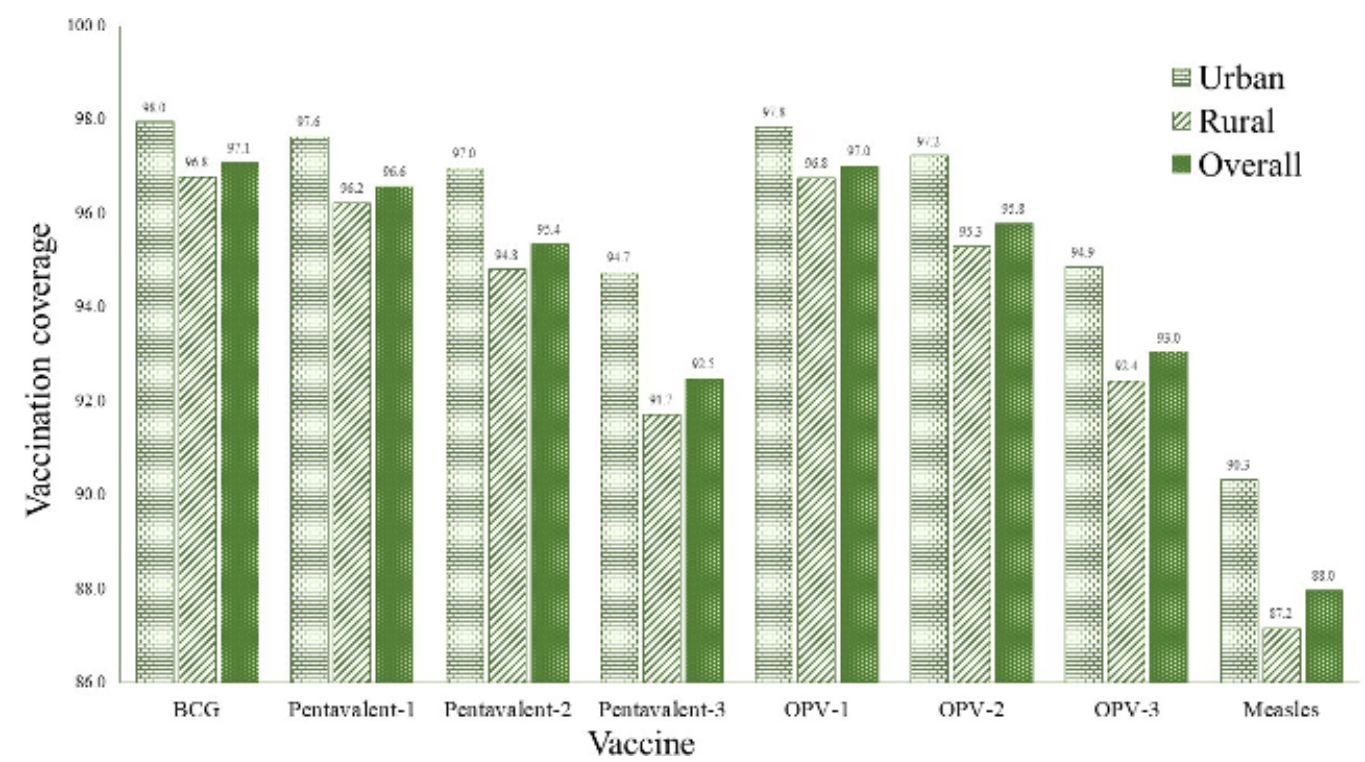

Figure 2 Coverage rate by individual vaccines across place of residence of respondents (urban vs rural) in Bangladesh. OPV, oral polio vaccine.

Full immunisation coverage was highest among the children whose parents had higher educational attainment $(94.2 \%$ and $93.7 \%$ for higher educated mother and father, respectively). Vaccine coverage rate was also higher among the children of mothers who have access to mass media. Full vaccination coverage was found to increase in accordance with a higher economic status of the children's family $(73.9 \%$ for the poorest to $93.2 \%$ for the richest quintile). Full immunisation coverage rate was higher among the children whose mother had her own healthcare decision-making ability, including that of her children's healthcare. The overall coverage rate of BCG vaccine was the highest $(97.1 \%)$ followed by OPV-1 (97\%), pentavalent 1 (96.6\%), OPV-2 (95.8\%) and pentavalent $2(95.4 \%)$ where the coverage of measles was the lowest $(88 \%)$ (figure 2). Furthermore, the coverage of each vaccine by urban and rural areas is also presented in figure 2.

\section{Factors associated with full immunisation coverage}

Table 3 shows factors associated with the full-immunisation coverage among children aged 12-59 months in terms of both unadjusted and AORs. The analysis shows that several sociodemographic factors, like administrative divisions, age of children, family size, parental education, wealth index and child healthcare decision-makers were associated with the full immunisation status in both models. From the univariate analysis, we found that the area of residence, childbirth order, access to mass media and mothers' healthcare decision-maker were significant factors for full immunisation. Urban children were significantly 1.35 times more likely to be fully vaccinated than rural ones (95\% CI 1.13 to $1.60, \mathrm{p}=0.001$ ), and the first-born child was 1.71 times more likely to be fully vaccinated than the child of third or more birth order $(95 \%$ CI 1.45 to $2.03, \mathrm{p}<0.001)$. We also observed that mothers of the children who had access to the mass media had higher odds than their counterparts $(\mathrm{OR}=2.01 ; 95 \% \mathrm{CI}$
1.72 to $2.36, \mathrm{p}<0.001)$. Similarly, parental education and wealth status played a significant role for full immunisation coverage among children. Findings revealed that children of higher educated mother were significantly 4.93 times (95\% CI 3.37 to $7.22, \mathrm{p}<0.001)$ and children of higher educated father were 4.03 times (95\% CI 2.98 to $5.44, \mathrm{p}<0.001)$ more likely to be fully immunised than children of uneducated mothers and fathers. Findings also revealed that children from the richest households were significantly 4.27 times (95\% CI 3.31 to $5.52, \mathrm{p}<0.001$ ) more likely to be fully immunised than the poorest.

In the adjusted model, the likelihood of being fully vaccinated was significantly lower in the Sylhet division than all other regions. We found that the number of full immunised children was significantly higher in Rangpur division $(\mathrm{AOR}=3.46 ; 95 \%$ CI 2.45 to $4.88, \mathrm{p}<0.001$ ) followed by the children from Dhaka (AOR $=2.59 ; 95 \% \mathrm{CI}$ 2.05 to $3.28, \mathrm{p}<0.001)$, Khulna (AOR $=2.33 ; 95 \%$ CI 1.62 to $3.33, \mathrm{p}<0.001)$ and Rajshahi $(\mathrm{AOR}=1.96 ; 95 \%$ CI 1.46 to 2.64, $\mathrm{p}<0.001$ ) division. The odds of full immunisation differed across age groups of the children. For instance, children aged 48-59 months had the highest odds of being fully vaccinated (AOR=1.32; 95\% CI 1.06 to 1.64, $\mathrm{p}=0.013$ ) compared with children aged 12-23 months. Family size was also appeared to be a significant factor for full immunisation as medium households (four to six members) often fully immunised more than their counterpart $(\mathrm{AOR}=1.56 ; 95 \%$ CI 1.32 to $1.86, \mathrm{p}<0.001)$. The findings also revealed that parental education was significantly associated with full immunisation. It was observed that the odds of being full immunisation were increased as the educational attainment of parents increased, and that children from higher educated mother $(\mathrm{AOR}=1.96$; 95\% CI 1.21 to $3.17, \mathrm{p}=0.006$ ) and higher educated father ( $\mathrm{AOR}=1.55 ; 95 \%$ CI 1.05 to $2.29, \mathrm{p}=0.026$ ) were more likely to be full vaccinated compared with those with 
Table 3 Unadjusted and adjusted effects of factors that are associated with full immunisation coverage

\section{Characteristics}

Area of residence

Urban

Rural

Division

$\begin{array}{lll}\text { Rajshahi } & 2.69(2.03 \text { to } 3.57) & <0.00 \\ \text { Barisal } & 2.43(1.73 \text { to } 3.41) & <0.001 \\ \text { Chittagong } & 2.53(2.01 \text { to } 3.18) & <0.001 \\ \text { Dhaka } & 3.43(2.76 \text { to } 4.26) & <0.00 \\ \text { Khulna } & 3.5(2.5 \text { to } 4.9) & <0.00 \\ \text { Rangpur } & 4.64(3.34 \text { to } 6.45) & <0.00 \\ \text { Sylhet } & 1.00 & \\ \text { Sex } & & \end{array}$

\begin{tabular}{|c|c|c|c|c|}
\hline Male & $0.93(0.81$ to 1.08$)$ & 0.342 & 0.92 (0.79 to 1.07$)$ & 0.294 \\
\hline Female & 1.00 & & 1.00 & \\
\hline
\end{tabular}

Age categories (in months)

\begin{tabular}{|c|c|c|c|c|}
\hline $12-23$ & 1.00 & & 1.00 & \\
\hline $24-35$ & 1.24 (1.02 to 1.51$)$ & 0.031 & 1.26 (1.02 to 1.56$)$ & 0.033 \\
\hline $36-47$ & 1.24 (1.01 to 1.51$)$ & 0.035 & 1.22 (0.99 to 1.52$)$ & 0.064 \\
\hline $48-59$ & 1.25 (1.02 to 1.52$)$ & 0.030 & 1.32 (1.06 to 1.64$)$ & 0.013 \\
\hline \multicolumn{5}{|l|}{ Birth order } \\
\hline 1 & 1.71 (1.45 to 2.03 ) & $<0.001$ & 1.16 (0.92 to 1.46$)$ & 0.204 \\
\hline 2 & $1.6(1.34$ to 1.91$)$ & $<0.001$ & 1.18 (0.96 to 1.45$)$ & 0.113 \\
\hline$\geq 3$ & 1.00 & & 1.00 & \\
\hline \multicolumn{5}{|l|}{ Mother's age } \\
\hline$<20$ years & 1.19 (0.86 to 1.64$)$ & 0.295 & 0.75 (0.5 to 1.12$)$ & 0.164 \\
\hline 20-34 years & 1.1 (0.86 to 1.42$)$ & 0.443 & 0.71 (0.53 to 0.95$)$ & 0.021 \\
\hline More than 35 years & 1.00 & & 1.00 & \\
\hline \multicolumn{5}{|l|}{ Family size (members) } \\
\hline Small $(<4)$ & 1.38 (1.09 to 1.75$)$ & 0.008 & 1.29 (0.97 to 1.72$)$ & 0.074 \\
\hline Medium (4-6) & 1.58 (1.36 to 1.85$)$ & $<0.001$ & 1.56 (1.32 to 1.86$)$ & $<0.001$ \\
\hline Large $(>6)$ & 1.00 & & 1.00 & \\
\hline \multicolumn{5}{|l|}{ Mother's education } \\
\hline No education & 1.00 & & 1.00 & \\
\hline Primary & 1.3 (1.08 to 1.57$)$ & 0.005 & 1.21 (0.98 to 1.49$)$ & 0.076 \\
\hline Secondary & 2.92 (2.42 to 3.54$)$ & $<0.001$ & 1.85 (1.45 to 2.35$)$ & $<0.001$ \\
\hline Higher & 4.93 (3.37 to 7.22$)$ & $<0.001$ & 1.96 (1.21 to 3.17$)$ & 0.006 \\
\hline \multicolumn{5}{|l|}{ Father's education } \\
\hline No education & 1.00 & & 1.00 & \\
\hline Primary & 1.52 (1.28 to 1.81$)$ & $<0.001$ & 1.18 (0.97 to 1.43$)$ & 0.099 \\
\hline Secondary & 2.5 (2.07 to 3.03) & $<0.001$ & 1.35 (1.06 to 1.71$)$ & 0.014 \\
\hline Higher & 4.03 (2.98 to 5.44$)$ & $<0.001$ & 1.55 (1.05 to 2.29$)$ & 0.026 \\
\hline \multicolumn{5}{|l|}{ Wealth index } \\
\hline Poorest & 1.00 & & 1.00 & \\
\hline Poorer & 1.82 (1.49 to 2.23$)$ & $<0.001$ & $1.41(1.13$ to 1.75$)$ & 0.002 \\
\hline Middle & 2.56 (2.05 to 3.18$)$ & $<0.001$ & 1.78 (1.37 to 2.3$)$ & $<0.001$ \\
\hline
\end{tabular}

Continued

\section{Adjusted OR $(95 \% \mathrm{Cl}) \quad P$ value}

$0.83(0.68$ to 1.03$) \quad 0.092$
1.00

$\begin{array}{ll}1.96(1.46 \text { to } 2.64) & <0.001 \\ 1.9(1.33 \text { to } 2.71) & <0.001 \\ 1.77(1.39 \text { to } 2.26) & <0.001 \\ 2.59(2.05 \text { to } 3.28) & <0.001 \\ 2.33(1.62 \text { to } 3.33) & <0.001 \\ 3.46(2.45 \text { to } 4.88) & <0.001 \\ 1.00 & \end{array}$




\begin{tabular}{|c|c|c|c|c|}
\hline Characteristics & Unadjusted OR (95\% Cl) & $P$ value & Adjusted OR $(95 \% \mathrm{Cl})$ & $P$ value \\
\hline Richer & 2.32 (1.88 to 2.86$)$ & $<0.001$ & 1.38 (1.03 to 1.84$)$ & 0.030 \\
\hline Richest & 4.27 (3.31 to 5.52$)$ & $<0.001$ & $2.2(1.5$ to 3.21$)$ & $<0.001$ \\
\hline \multicolumn{5}{|l|}{ Access to mass media } \\
\hline Yes & 2.01 (1.72 to 2.36$)$ & $<0.001$ & 1.15 (0.92 to 1.43$)$ & 0.214 \\
\hline No & 1.00 & & 1.00 & \\
\hline \multicolumn{5}{|l|}{$\begin{array}{l}\text { Mothers healthcare } \\
\text { decision-maker }\end{array}$} \\
\hline Herself & 1.00 & & 1.00 & \\
\hline Jointly with husband & 0.88 (0.68 to 1.13$)$ & 0.312 & 0.89 (0.66 to 1.2$)$ & 0.431 \\
\hline Husband alone & 0.54 (0.42 to 0.7$)$ & $<0.001$ & 0.77 (0.57 to 1.06$)$ & 0.107 \\
\hline By other & 0.76 (0.52 to 1.1$)$ & 0.146 & 0.67 (0.42 to 1.08$)$ & 0.098 \\
\hline \multicolumn{5}{|c|}{$\begin{array}{l}\text { Child healthcare decision- } \\
\text { maker }\end{array}$} \\
\hline Herself & 1.00 & & 1.00 & \\
\hline Jointly with husband & 0.99 (0.79 to 1.23$)$ & 0.910 & 1.06 (0.82 to 1.39$)$ & 0.641 \\
\hline Husband alone & 0.51 (0.4 to 0.65$)$ & $<0.001$ & 0.69 (0.51 to 0.92$)$ & 0.012 \\
\hline By other & $1.02(0.7$ to 1.49$)$ & 0.912 & 1.3 (0.81 to 2.07$)$ & 0.277 \\
\hline Mean VIF & & & 3.07 & \\
\hline $\operatorname{LR} \chi^{2}$ & & & 438.82 & \\
\hline Prob $>\chi^{2}$ & & & $<0.001$ & \\
\hline Pseudo $\mathrm{R}^{2}$ & & & 0.089 & \\
\hline \multicolumn{2}{|c|}{ Linear predicted value (_hat) } & & $<0.001$ & \\
\hline \multicolumn{2}{|c|}{ Linear predicted value squared (_hatsq) } & & 0.885 & \\
\hline \multicolumn{2}{|c|}{$\mathrm{HL} \chi^{2}(8)$} & & 7.88 & \\
\hline \multicolumn{2}{|l|}{ Prob $>\chi^{2}$} & & 0.4452 & \\
\hline
\end{tabular}

HL, Hosmer-Lemeshow; LR, likelihood ratio; VIF, variance inflation factor.

uneducated parents. Children from the richest households had the highest odds of being fully immunised $(\mathrm{AOR}=2.20 ; 95 \%$ CI 1.50 to $3.21, \mathrm{p}<0.001)$ followed by the middle wealth quintile $(\mathrm{AOR}=1.78 ; 95 \%$ CI 1.37 to $2.30, \mathrm{p}<0.001)$. The lowest odds were found among the children whose healthcare decision was made solely by their father (AOR $=0.69 ; 95 \%$ CI 0.51 to $0.92, p=0.012$ ) compared with children with healthcare decisions that were made by the mothers.

\section{DISCUSSION}

The present study examined the extent of full immunisation coverage and determinants of full immunisation status. We found that the overall full immunisation coverage is impressive (86\%) within South East Asia Region..$^{25}$ The underlying success is due to the nature of a pluralistic health system of Bangladesh, where the public, private sector and non-governmental organisations actively participate to deliver healthcare services. In addition, the introduction of systematic outreach approaches helped to increase accessibility to routine immunisation where community health workers are directly involved in delivering vaccines. ${ }^{26}$ Further, community mobilisation and public awareness-related programmes have also played a significant role in generating demand for vaccine uptake. ${ }^{27}$

Despite the success, full immunisation coverage varies across the country. For instance, the full immunisation coverage is the highest in Rangpur while the lowest coverage is observed in the Sylhet region. Our study showed that various factors, such as parental education particularly mothers' education, age of the children, family size, regional variation and wealth quintiles played a significant role for full immunisation coverage. The importance of parental education in improving child's health is universally recognised. Similar to earlier studies on childhood vaccination, we found that parental education appeared to be a significant factor for childhood immunisation. ${ }^{14} 28$ This is due to their better knowledge and understanding of the recommended immunisation schedules than non-educated parents. ${ }^{29}$ Furthermore, educated parents are likely to be wealthier and have better access to health facilities and immunisation services. ${ }^{28}$ Like other studies in various settings, we 
observed that the children of educated mothers were often fully immunised when compared with non-educated mothers. ${ }^{1428}{ }^{30}$ It is well established that there is a positive relationship between the level of education and public health awareness. Therefore, community-based behaviour changes programmes, such as immunisation announcement through radio, television and using local drama and public announcement through miking (loud speaker), should be approached to target uneducated mothers in order to provide a better understanding of the beneficial role of immunisation so that they are encouraged to vaccinate their children in a timely fashion.

We observed that full immunisation coverage was higher among elder children (eg, 48-59 months of age) compared with the younger children (eg, 12-23 months of age). Policy-makers should provide more focus on continuous monitoring and promotional activities to increase the number of full immunisation coverage of all strata. ${ }^{14}$ Again, the size of the family appeared to be a significant factor of fully immunised children. Those who belonged to larger family size were more likely to be unimmunised. This is similar to the findings in other earlier studies conducted in Indonesia, Greece and Angola, where it was also reported that children form larger family size were less likely to be fully immunised. ${ }^{14} 3132$ This might also reflect the socioeconomic status of the households as larger families consume more resources, which is troublesome for resource-poor families and would thus impact on their livelihoods. Therefore, policies should focus on awareness development, especially in targeting the larger family to increase the full immunisation coverage. Wealth status of the household plays a significant role in the immunising of their children. Our results are similar to many earlier studies which have shown that there is a significant positive relationship between wealth index and childhood immunisation completion rates; the chance of having fully immunised children increases in accordance with the wealth index of household. ${ }^{14}{ }^{2830}$ While the immunisation services in Bangladesh are completely free of charge, the indirect cost of vaccination, such as income loss and transportation cost, might be associated with the low demand for vaccination specially for poorest households. ${ }^{334}$ Children from urban areas have been reported to have better immunisation status compared with their rural counterparts. This result confirmed previous findings where similar association was also reported. ${ }^{1435}$ This is due to better health services available in urban areas compared with the rural. ${ }^{36}$

Our study observed that child healthcare decision-making by mothers also contributed, playing a significant role in full immunisation coverage, with the child more likely to be fully immunised if the mother was the decision-maker. This might be due to more awareness of the mothers for their child's health than the fathers. Therefore, the mothers' autonomy is essential for their child's immunisation. However, an earlier study showed that if the parents jointly decided on healthcare decision, their children were often fully immunised. ${ }^{14}$ Thus, there is a need to disseminate information about women autonomy in making healthcare decisions about their children to increase full immunisation coverage. Based on the findings, community-based behaviour change programme that targets parents might be helpful for developing awareness for their childhood immunisation. Like many low-income and middle-income countries, we also observed that geographical disparities may contribute to the full immunisation coverage in Bangladesh. ${ }^{1437-39}$ Our study found that children who lived in Sylhet region were more often unimmunised when compared with other parts of the country. This is may be due to various supply-side and demand-side factors, such as the distance of health facilities and vaccination centres, fragile communication systems in some remote areas, a fear of the side effects of vaccines, religious conservatism, a low level of literacy and even lack of awareness about the benefit of vaccination for their children. ${ }^{39}$ Therefore, policy should target divisions with low immunisation coverage with an innovative immunisation approach that addresses both supply-side and demand-side barriers. For instance, households that are located away from or have difficulty in accessing immunisation services, especially in hilly areas, might benefit from outreach programme or mobile immunisation strategies. Furthermore, the use of mobile phones may be important vehicles for tracking and improving immunisation coverage in these rural, hard to reach areas of Bangladesh. ${ }^{40}$

\section{Strengths and limitations}

The study has several limitations. The study was based on secondary data where immunisation cards and mother's report were considered as the source of information for their child's immunisation status. Therefore, the potential of recall bias in our results cannot be ignored. Nonetheless, the mother's report is considered to be a valid measure of coverage in the absence of a health card, especially in developing countries. ${ }^{41}$ The explanatory variables were selected based on previous studies and relied on the information available from the dataset. Therefore, there might be some other potential predictors that might be influenced by full immunisation which were not captured in this study. Despite the limitations, the study results can be generalised at a country level because the study used data from the latest nationally representative household survey. Thus, our findings are still significant and relevant in drawing attention to the health policy-makers in ensuring the benefit of vaccination for the betterment of child health.

\section{CONCLUSION}

Our results identified the presence of disparities for full immunisation coverage across regions and by types of vaccines in Bangladesh. Findings revealed that full immunisation status was significantly associated with regional variation, the age of the children, maternal age, parental education, family size, woman autonomy for 
child healthcare and wealth quintiles. Our study found that a large number of children from the Sylhet division, poor and larger family, and from lower parental education were not fully immunised. The study also identified that measles immunisation coverage was the lowest among the eight recommended vaccines. Therefore, to achieve maximum success and prevent children from VPD, it is mandatory to address the issues obstructing full vaccination. The findings of this study will serve to inform and support innovative approach for immunisation programmes, and the introduction of relevant policies, including the regular monitoring and evaluation of immunisation coverage particularly for low-performing regions of Bangladesh and targeting various vaccines in order to allow the broader benefit of the immunisation programme to be achieved in all strata of society.

Acknowledgements ICDDRB is thankful to the Governments of Australia, Bangladesh, Canada, Sweden and the UK for providing core/unrestricted support. The authors would like to thank Health Economics and Financing Research Group of ICDDRB for their earlier comments in this research. The authors acknowledges with gratitude the commitment of Bangladesh Institute of Development Studies to its research efforts.

Contributors ARS and MS participated in the design of the study. All authors performed the analysis and prepared the manuscript. ARS, RA and MS provided data analysis advice and revision of the manuscript. All authors read and approved the final manuscript.

Funding The authors have not declared a specific grant for this research from any funding agency in the public, commercial or not-for-profit sectors.

Map disclaimer The depiction of boundaries on the map(s) in this article do not imply the expression of any opinion whatsoever on the part of BMJ (or any member of its group) concerning the legal status of any country, territory, jurisdiction or area or of its authorities. The map(s) are provided without any warranty of any kind, either express or implied.

Competing interests None declared.

Ethics approval This study did not require ethical approval as it used unidentifiable secondary DHS dataset. According to the DHS, written informed consent was obtained from mothers/caretakers on behalf of the children enrolled in the survey. The DHS data are publicly accessible and were made available to us upon request by Measure DHS. No identifiable information was included in the dataset and no attempt was made to identify any individual interviewed in the survey.

Provenance and peer review Not commissioned; externally peer reviewed.

Data sharing statement The electronic datasets analysed in this study are available for legitimate research purposes from the Measure DHS website (https:// dhsprogram.com/data/).

Open access This is an open access article distributed in accordance with the Creative Commons Attribution Non Commercial (CC BY-NC 4.0) license, which permits others to distribute, remix, adapt, build upon this work non-commercially, and license their derivative works on different terms, provided the original work is properly cited, appropriate credit is given, any changes made indicated, and the use is non-commercial. See: http://creativecommons.org/licenses/by-nc/4.0/.

\section{REFERENCES}

1. World Health Organization (WHO). Protecting people from Vaccine Preventable Diseases: Strategic Plan (2010-2013). Geneva 27, Switzerland 2013.

2. Andre FE, Booy R, Bock HL, et al. Vaccination greatly reduces disease, disability, death and inequity worldwide. Bull World Health Organ 2008;86:140-6.

3. Unicef WHO, Bank W. State of the World's Vaccine and Immunization. 3rd ed. Geneva: World Health Organization (WHO), 2009.
4. Jamil K, Bhuiya A, Streatfield K, et al. The immunization programme in Bangladesh: impressive gains in coverage, but gaps remain. Health Policy Plan 1999;14:49-58.

5. Keja K, Chan C, Hayden G, et al. Expanded programme on immunization. World Heal Stat Q 1988;41:59-63.

6. Quaiyum MA, Tuñon C, Hel Baqui A, et al. Impact of national immunization days on polio-related knowledge and practice of urban women in Bangladesh. Health Policy Plan 1997;12:363-71.

7. Sarkar PK, Sarker NK, Doulah S, et al. Expanded Programme on Immunization in Bangladesh: A Success Story. Bangladesh Journal of Child Health 2015;39:93-8.

8. Mik S, Mf S, Kurlikar PR, et al. Status and determinants of child immunisation coverage in three South Asian countries, India, Bangladesh and Nepal : Evidence from the Demographic and Health Survey. Sri Lanka J Child Heal 2018;47:56-63.

9. Expanded Program on Immunization (EPI). Bangladesh EPI Coverage Evaluation Survey 2016. Dhaka, Bangladesh 2017.

10. United Nations Inter-agency Group for Child Mortality Estimation (UN IGME). Levels \& Trends in Child Mortality: Report 2017, Estimates Developed by the UN Inter-agency Group for Child Mortality Estimation. New York 2017.

11. Liu L, Oza S, Hogan D, et al. Global, regional, and national causes of child mortality in 2000-13, with projections to inform post-2015 priorities: an updated systematic analysis. Lancet 2015;385:430-40.

12. Rahman M, Obaida-Nasrin S. Factors affecting acceptance of complete immunization coverage of children under five years in rural Bangladesh. Salud Publica Mex 2010;52:134-40.

13. Herliana P, Douiri A. Determinants of immunisation coverage of children aged 12-59 months in Indonesia: a cross-sectional study. BMJ Open 2017;7:1-14.

14. Oleribe O, Kumar V, Awosika-Olumo A, et al. Individual and socioeconomic factors associated with childhood immunization coverage in Nigeria. Pan Afr Med J 2017;26:1-14.

15. Meleko A, Geremew M, Birhanu F. Assessment of Child Immunization Coverage and Associated Factors with Full Vaccination among Children Aged 12-23 Months at Mizan Aman Town, Bench Maji Zone, Southwest Ethiopia. Int J Pediatr 2017;2017:1-11.

16. Sheikh N, Sultana M, Ali N, et al. Coverage, Timelines, and Determinants of Incomplete Immunization in Bangladesh. Trop Med Infect Dis 2018;3:1-14.

17. Uddin MJ, Larson CP, Oliveras E, et al. Child immunization coverage in urban slums of Bangladesh: impact of an intervention package. Health Policy Plan 2010;25:50-60.

18. Rahman A, Reza AAS, Bhuiyan BA, et al. Equity and determinants of routine child immunisation programme among tribal and non-tribal populations in rural Tangail subdistrict, Bangladesh: a cohort study. BMJ Open 2018;8:e022634.

19. Adhikary M, Haque R, Tanira S. Determinants of child immunization under expanded programme on immunization (EPI) in a rural setting of Bangladesh. J Dhaka Med Coll 2013;22:201-6.

20. Nasrin Afzal BZ. Determinants and Status of Vaccination in Bangladesh. Dhaka UnivJSci 2012;60:47-51.

21. Rutstein SO, Rojas G. Guide to DHS statistics: Demographic and Health Surveys Methodology. Calverton, Maryland 2006.

22. National Institute of Population Research and Training (NIPORT), Mitra and Associates and II 2016. Bangladesh Demographic and Health Survey 2014. Dhaka, Bangladesh, and Rockville, Maryland, USA: NIPORT, Mitra and Associates, and ICF International, 2014.

23. Directorate General of Health Services (DGHS). EPI vaccine Schedule. Minist. Heal. Fam. Welf. http://www.dghs.gov.bd/index. php/en/mis-docs/epi (Accessed 10 Mar 2019).

24. WHO. WHO South-East Asia:Expanded Programme on Immunization (EPI) REGIONAL FACT SHEET 2017. World Heal Organ 2017;6:1-98.

25. Adams AM, Rabbani A, Ahmed S, et al. Bangladesh: Innovation for Universal Health Coverage 4: Explaining equity gains in child survival in Bangladesh: Scale, speed, and selectivity in health and development. Lancet 2013.

26. El Arifeen S, Christou A, Reichenbach L, et al. Community-based approaches and partnerships: innovations in health-service delivery in Bangladesh. Lancet 2013;382.

27. Holipah, Maharani A, Kuroda Y. Determinants of immunization status among 12- to 23-month-old children in Indonesia (2008-2013): a multilevel analysis. BMC Public Health 2018;18:1-11.

28. Schoeps A, Ouédraogo N, Kagoné M, et al. Socio-demographic determinants of timely adherence to BCG, Penta3, measles, and complete vaccination schedule in Burkina Faso. Vaccine 2013;32

29. Abadura SA, Lerebo WT, Kulkarni U, et al. Individual and community level determinants of childhood full immunization in Ethiopia: A multilevel analysis Global health. BMC Public Health 2015;15:1-10.

30. Danis K, Georgakopoulou T, Stavrou T, et al. Socioeconomic factors play a more important role in childhood vaccination coverage than 
parental perceptions: a cross-sectional study in Greece. Vaccine 2010;28:1861-9.

31. Oliveira MF, Martinez EZ, Rocha JS. Factors associated with vaccination coverage in children $<5$ years in Angola. Rev Saude Publica 2014;48:906-15.

32. Mitchell S, Andersson N, Ansari NM, et al. Equity and vaccine uptake: a cross-sectional study of measles vaccination in Lasbela District, Pakistan. BMC Int Health Hum Rights 2009;9 Suppl 1:S7.

33. Lanaspa M, Balcells R, Sacoor C, et al. The performance of the expanded programme on immunization in a rural area of Mozambique. Acta Trop 2015;149.

34. Shrivastwa N, Gillespie BW, Kolenic GE, et al. Predictors of Vaccination in India for Children Aged 12-36 Months. Am J Prev Med 2015;49.

35. Fernandez R, Rammohan A, Awofeso N. Correlates of first dose of measles vaccination delivery and uptake in Indonesia. Asian Pac $J$ Trop Med 2011;4:140-5.

36. Clouston S, Kidman R, Palermo T. Social inequalities in vaccination uptake among children aged 0-59 months living in Madagascar: an analysis of Demographic and Health Survey data from 2008 to 2009 Vaccine 2014;32.

37. Antai D. Inequitable childhood immunization uptake in Nigeria: a multilevel analysis of individual and contextual determinants. BMC Infect Dis 2009:9:181.

38. Uddin MJ, Koehlmoos TP, Saha NC, et al. Child immunization coverage in rural hard-to-reach areas of Bangladesh. Vaccine 2010;28:1221-5.

39. Uddin MJ, Shamsuzzaman M, Horng L, et al. Use of mobile phones for improving vaccination coverage among children living in rural hard-to-reach areas and urban streets of Bangladesh. Vaccine 2016;34.

40. Langsten R, Hill K. The accuracy of mothers' reports of child vaccination: evidence from rural Egypt. Soc Sci Med 1998;46:120512.

41. Boulton ML, Carlson BF, Power LE, et al. Socioeconomic factors associated with full childhood vaccination in Bangladesh, 2014. Int $J$ Infect Dis 2018;69:35-40. 